\title{
Fremdadoptionsrecht auch für homosexuelle Lebenspartnerschaften?
}

\section{Hinweise auf bislang nicht beachtete Herausforderungen}

\section{Rosemarie Nave-Herz}

Zusammenfassung: Im letzten Heft wurde die Rubrik „Zwischenruf“ eingeführt. Sie befasst sich mit laufend aktuellen Bezüge zu gesellschaftlichen Themen und diskutiert diese aus individueller Perspektive. Der aktuelle „Zwischenruf“ behandelt das Fremdadoptionsrecht homosexueller Lebenspartnerschaften. Die Auswirkungen von Adoption auf Kinder werden mit kritischen Nachfragen einer solchen Erweiterung beleuchtet.

Schlüsselwörter: Fremdadoptionsrecht · Homosexuelle Lebenspartnerschaften · Sozialisationsverläufe

\section{Adoption for homosexual life-partnerships, too?}

\begin{abstract}
In the last journal the column "Zwischenruf" was presented. It deals with current social topics, which are discussed and reflected from an individual perspective. The new "Zwischenruf" discusses the possibility of adoption for homosexual couples. It asks critically about consequences for children.
\end{abstract}

Keywords: Adoption · Homosexual Life-partnerships $\cdot$ Course of socialization

(C) Die Autor(en) 2012. Dieser Artikel ist auf Springerlink.com mit Open Access verfügbar.

Prof. Dr. Dr. h. c. R. Nave-Herz $(\bowtie)$

Fak. 1/Institut für Sozialwissenschaften, C.v.O.-Universität, Ammerländer Heerstr. 114, 26129 Oldenburg, Deutschland

E-Mail: rosemarie.nave.herz@uni-oldenburg.de 
Erst die Studentenbewegung - vor ca. 40 Jahren - bereitete den Weg zu einer beginnenden öffentlichen Meinungsveränderung über Homosexualität, weil sich nunmehr die Homosexuellen selbst von ihrer bisher passiven Rolle lösten. Vor allem im Rahmen der Frauenbewegung betonten erstmals lesbische Frauen ihre sexuelle Orientierung als positiv, als Befreiungsmöglichkeit. In den 1970er Jahren entstanden die ersten Schwulengruppen, die aus ihrer „Heimlichkeit“ heraus an die Öffentlichkeit traten und das Recht forderten, offen so zu sein, wie sie sind. Die Homosexuellen unterstrichen ihre Normalität. Sie ,stellten sich gegen den Rest der Gesellschaft und forderten ihren Platz an der Sonne“" (Ariés 1984, S. 85).

Bereits zuvor kündigten die rechtlichen Veränderungen, vor allem durch die gesetzliche Lockerung des $§ 175$ StGB (1969), der Homosexualität für Männer als Straftatbestand definierte (auf lesbische Frauen bezog sich der $§ 175$ StGB nicht) und dann 1994 durch seine Abschaffung symbolisch die neuen Phasen in der Bedeutungszuschreibung von Homosexualität an. Denn damit wurde eine Ausgangsbasis geschaffen, von der aus Homosexualität und homosexuelle Partnerschaft als eigenständige Lebensform überhaupt definierbar, anerkannt und lebbar wurden. Seit dem 16. 2. 2001 (erweitert 2005) haben homosexuelle Paare die Möglichkeit, eine „Eingetragene Lebenspartnerschaft“ $\mathrm{zu}$ begründen und damit eherechtliche Ansprüche zu erwerben, in Bezug auf Erbschafts- und Versorgungsansprüche, im Beamtenrecht, im Mietrecht u.a.m. (vgl. Tann 2012). Aber noch immer sind Unterschiede zwischen Ehen und Eingetragenen Lebenspartnerschaften gegeben, was von den Betroffenen als Diskriminierung empfunden wird. Auch deren Abschaffung wird zurzeit von ihnen und von parteipolitischen Gruppierungen gefordert. Dazu gehört die Forderung nach Fremdadoption von Kindern. Warum nicht?

Es ist verständlich, dass auch Homosexuelle - wie alle Menschen - das Bedürfnis haben, Kinder bei sich aufwachsen zu sehen, von ihnen geliebt zu werden, überhaupt die expressive Stimulation, die von ihnen ausgeht, zu erleben, ihre Lebenserfahrungen u.a.m. an die nächste Generation unmittelbar weiter zu vermitteln. Die Adoption von Kindern des Partners oder der Partnerin ist bereits als Rechtsanspruch formuliert. Im Übrigen sind diese „,immateriellen Werte von Kindern“ für hetero-sexuelle Frauen und Männer heutzutage in allen Industriestaaten für die Einlösung ihres Kindeswunsches ebenso ausschlaggebend geworden, wie die Ergebnisse der Value-of-children-Forschung zeigen (vgl. Nave-Herz 2012).

Soziologisch kann man von einer „Instrumentalisierung von Kindern“ sprechen. Wie in der vorindustriellen Zeit, als Kinder wegen ihrer Arbeitskraft, als Helfer im Krankheitsfall und im Alter, zur Weitergabe des familialen Vermögens und des Namens gewünscht und erhofft wurden, werden auch heute Kinder nicht allein um ihrer selbst willen ,geplant“, sondern um einen persönlichen Gewinn zu erzielen. Bei Fremdadoptionen durch homosexuelle Partner wird nunmehr diese Bedeutung von Kindern öffentlich als Forderung formuliert (vgl. z. B. Emcke 2012). Stehen solche Elterninteressen und die Erfüllung ihrer Bedürfnisse nicht evtl. in einem Spannungsfeld zu dem - heute allgemein anerkannten - Postulats des Kinderwohls?

Bei gleichgeschlechtlichen Paaren mit Kindern stammen diese zumeist aus einer früheren Ehe oder einer nicht-ehelichen Partnerschaft; überwiegend handelt es sich um lesbische Paare (vgl. Vascovics und Buba 2000; Eggen 2002). Nach Maier (2009) sollen von allen Kindern in Deutschland ca. 13000 in gleichgeschlechtlichen Lebensformen 
heranwachsen. Doch gleichzeitig betont die Autorin die Schwierigkeit der statistischen Erfassung und zitiert weit von ihrer Zahl abweichende Daten. In einer Erhebung von Rupp (2009) gaben 268 von 1.059 homosexuellen Paaren mit Kindern an, dass das Kind durch Insemination gezeugt worden war. Es wird vermutet, dass sich verstärkt in Zukunft, nämlich bei gestiegener Akzeptanz von homosexuellen Eltern, lesbische Frauen für eine Insemination im Ausland entscheiden, ein Vorzug, der für Männer, die sich Kinder wünschen, nicht infrage kommt.

Auch wenn die Befunde zu gleichgeschlechtlichen Eltern mit Kindern rar sind, keine empirische Untersuchung und keine Kriminalitätsstatistik belegt, dass homosexuelle Eltern „schlechtere Eltern“ - was immer darunter zu verstehen ist - wären als heterosexuelle. Psychologische Veröffentlichungen, demographische Analysen, ferner Betroffenheitsliteratur oder Schriften, herausgegeben von den verschiedenen homosexuellen Bewegungen dominieren. Die wenigen in Deutschland vorhandenen Untersuchungen sowie die ausländischen Forschungsergebnisse beziehen sich nicht auf Fremdadoptionen. Sie sind zudem häufig wegen ihrer kleinen und selektiven Stichprobe nicht unbedingt verallgemeinerungsfähig. Aus ihnen ist übereinstimmend zu entnehmen, dass letztlich in Bezug auf diese betroffenen Kinder keine stärkeren Sozialisationsdefizite festzustellen seien als in anderen Familienformen. Nicht die familiale Struktur beeinflusst allein oder deterministisch das Gelingen des kindlichen Entwicklungsprozesses, sondern ausschlaggebend sind für die Sozialisation der Kinder die Qualität der familialen Beziehungen, die häusliche Atmosphäre, der Lebensstil, das Gefühl seitens des Kindes, angenommen und geborgen $\mathrm{zu}$ sein, das Anregungspotenzial. Warum also kein Anspruch auf eine Fremdadoption?

Dennoch ist zu bedenken, dass die Kinder in dieser Familienform, selbst also diejenigen mit einer biologischen Abstammung von einem Elternteil, sich in einer sehr schwierigen gesellschaftlichen Ausnahme- und Randposition befinden, die ihnen spezifische Bewältigungsstrategien abverlangt. In der Studie von Rupp (2009) berichten die befragten Kinder mit einem gleichgeschlechtlichen Elternteil zu 46\% von erfahrenen diskriminierenden Erlebnissen. Am häufigsten werden Hänseleien und Beschimpfungen genannt. Die Vorfälle finden zumeist im schulischen Bereich statt, doch ein Zehntel der betroffenen Kinder hat die entsprechenden Erfahrungen bereits im Kindergarten gemacht. Sie erleben ferner vielfach die Stigmatisierung ihrer Eltern. Denn über Diskriminierungserfahrungen berichten homosexuelle Paare in vielen Erhebungen und Fallbeispielen, z. B. auf dem Wohnungsmarkt, am Arbeitsplatz, von der Herkunftsfamilie usw. (vgl. Steffens 2010). Wegen dieser psychisch starken Belastung durch die Vorurteile gegenüber Homosexuellen in der Bevölkerung wurden nicht zuletzt von ihren Verbänden die Gesetzesänderungen gefordert. Doch alle Rechtssoziologen würden der Hoffnung, durch Gesetzesänderungen einen Einstellungswandel zu erreichen, eine Absage erteilen. Gesetze wirken auf gesellschaftliches Bewusstsein nicht verändernd; gerade aus dem Familienrecht gibt es hierzu zahlreiche Belege. Diese Erfahrungen haben auch die Frauen gemacht, denen bereits im Grundgesetz gleiche Rechte und die soziale Gleichstellung in allen gesellschaftlichen Teilbereichen versprochen wurde. Aber erst die Neue Frauenbewegung hat den ersten Anstoß zur gesellschaftlichen Veränderung vor allem auf der - zunächst notwendigen, aber nicht ausreichenden - Bewusstseinsebene bewirkt. 
Von den Kindern in homosexuellen Partnerschaften wird ferner verlangt, sich gegen das Vorurteil ihnen gegenüber zu wehren, nämlich selbst prädestiniert für Homosexualität zu sein. Doch wie viele empirische Untersuchungen aus den USA zeigen, ist die - in der Öffentlichkeit häufig vermutete und geäußerte - Gefahr, durch das Aufwachsen von Kindern in homosexuellen Partnerschaften selbst homosexuell zu werden, kaum gegeben. Die sexuelle Orientierung ihrer Eltern soll also (vgl. Savin-Williams und Esterberg 2000; Fthenakis und Ladwig 2002; Streib-Brzic und Gerlach 2005; Maier 2009) keinen Einfluss auf die Entwicklung der Geschlechtsidentität nehmen. Das schließt nicht aus, dass sich dennoch diese Kinder durch die Konfrontation mit der bei uns noch immer als Stigma empfundenen sexuellen ,Andersartigkeit“ ihrer Eltern und durch die eigene Entstehungsgeschichte dieser Familien in ihren Sozialisationsverläufen von anderen unterscheiden und vor allem - wie bereits betont - spezifische Bewältigungsstrategien von ihnen abverlangt werden.

Die biologischen Eltern - ebenso wie die Geschwister - haben den „Charakter des Schicksalhaften“: man kann sie sich nicht aussuchen. Diese Schicksalshaftigkeit fehlt generell bei der Fremdadoption, bei gleich- wie bei andersgeschlechtlichen. Zwischen biologischen Eltern und ihren Kindern entsteht zumeist bereits während der Schwangerschaft ein emotionales Band, und das vereinigt bzw. verbindet sie - trotz Krisen, Konflikten evtl. auch mit gegenseitigen Verletzungen, bei Trennungen - aus welchen Gründen auch immer - ein Leben lang, wie in der Psychologie nachgewiesen wurde. Selbst nach dem Coming Out eines Elternteiles bleibt es zumeist - evtl. nach starken „Erschütterungen“" - bestehen, vor allem auch dann, wenn das Zusammenleben mit dem homosexuellen Elternteil durch freie Entscheidung überdauerte. Für den individuellen Verarbeitungsprozess und gegenüber der „Außenwelt" beinhaltet dieses seit Geburt bestehende emotionale Band eine nachhaltige psychische Unterstützung junger Menschen. Fremdadoptierten Kindern durch homosexuelle Paare fehlt diese „Schicksalsverbundenheit“. Wie die durch künstliche Befruchtung gezeugten Kinder bei Kenntnis des Familienbildungsprozesses die „fehlende Familiengeschichte“, die Unkenntnis ihrer ,Wurzeln“, jedenfalls in Bezug auf den biologischen Vater, psychisch verarbeiten, ist empirisch eine offene Frage; lediglich spekulative Erörterungen oder Einzelfallstudien wurden bisher verfasst. Fremdadoptierte Kinder von homosexuellen Paaren wachsen zudem noch mit den - aus der Adoptionsforschung bekannten - spezifischen innen- und außerfamilialen Beziehungsproblemen auf, wie sie bereits 1989 von Hoffmann-Riem (1989, S. 397)ausführlich beschrieben wurden. Im Hinblick auf diese doppelte Ausnahmeposition, in der sich diese Kinder befinden werden, stellt sich wiederum die Frage nach der Einlösung des Postulats des Kinderwohls bei der Entscheidung, homosexuellen Partnern evtl. das Fremdadoptionsrecht zu gewähren.

Der Begriff „Kindeswohl“ wurde in den letzten Jahrzehnten zu einem leitenden Rechtsbegriff, der internationale Geltung beansprucht. Das Kindeswohl (best interest of the child) gehört auch zu den zentralen Begriffen der Kinderrechtskommission der Vereinten Nationen: in Art. 3 Abs. 1 KRK ist die Vorrangigkeit des Kindeswohls festgeschrieben. Mit ihm hat man einen Perspektivenwechsel in der Rechtsprechung dokumentiert: weg von der alleinigen elterlichen Sorge um das Kind hin zur Wahrung der Interessen des Kindes, d.h. das, was für das Kind selbst zum Wohle ist, soll gelten. Dennoch bleibt der Begriff juristisch unbestimmt; es müssen deshalb unter Berücksichtigung individueller Bedingungen Einzelfall-Entscheidungen getroffen werden. Rechtswissenschaftler (vgl. 
Münder 2005; Wiesner 2011) betonen, dass mit dem Postulat des Kindeswohls zum einen die Interventionspflicht des Staates legitimiert wird, um weitere Kindesgefährdung zu vermeiden. Dabei ist vor allem primäres Ziel, für Eltern zum Wohle des Kindes unter Berücksichtigung der individuellen Bedingungen Unterstützung in Form von problemangemessenen Hilfen zu entwickeln und ihnen zu bieten. Zum anderen bezieht sich juristisch das Postulat des Kindeswohls auch auf Prävention, nämlich Kindesgefährdung zu vermeiden. Die Fremdadoption durch homosexuelle Paare kann wohl nicht - wie gezeigt - im juristischen Sinne als eine Kindesgefährdung definiert werden.

In den Sozialwissenschaften und der Psychologie gibt es zwar verschiedene Definitionen von „Kindeswohl“ und „Kindesgefährdung“. Sie gehen aber alle über die engen Grenzen der juristischen Begriffsinterpretation hinaus. Sie knüpfen stärker - wie schon im Art. 3 Abs. 1 KRK - an die Bedürfnisse der Kinder an und betonen übereinstimmend - wenn auch mit unterschiedlicher Akzentuierung - die Förderung und die Hilfe zur Entwicklung von Kindern.

Müsste nicht unter dieser normativen Perspektive, der Parteinahme für die Kinder, und wegen der Gefahr einer fehlenden schicksalhaften Verbundenheit der Kinder mit ihren ,neuen“ Eltern die Frage nach dem Fremdadoptionsrecht durch homosexuelle Paare skeptischer betrachtet werden, als das zuweilen der Fall ist? Aus der Perspektive der Kinder argumentiert, ginge es dann darum, ihnen die doppelte Rand- und Ausnahmeposition mit ihren spezifischen Bewältigungsproblemen zu ersparen. In der gegenwärtigen Diskussion sollten die hier vorgetragenen Argumente zumindest prominenter als bislang Beachtung finden. Neue Formen der Diskriminierung und der Instrumentalisierung von Kindern sollten auf jeden Fall verhindert werden.

Open Access Dieser Artikel unterliegt den Bedingungen der Creative Commons Attribution License. Dadurch sind die Nutzung, Verteilung, und Reproduktion erlaubt, sofern der/die Originalautor/en und die Quelle angegeben sind.

\section{Literatur}

Ariés, P. (1984). Überlegungen zur Geschichte der Homosexualität. In P. Ariés et al. (Hrsg.), Die Masken des Begehrens und die Metamorphosen der Sinnlichkeit - Zur Geschichte der Sexualität im Abendland (S. 80-96). Frankfurt: Fischer.

Eggen, B. (2002). Gleichgeschlechtliche Lebensgemeinschaften. Erste Ergebnisse einer Untersuchung im Rahmen des Mikrozensus. In N. F. Schneider \& H. Matthias-Bleck (Hrsg.), Elternschaft heute (S. 215-234). Opladen: Leske \& Budrich.

Emcke, C. (2012). Ich bin es leid. Die Zeit, 34, 3.

Fthetnakis, W. E., \& Ludwig, A. (2002). Homosexuelle Väter. In W. E. Fthenakis \& M. R. Textor (Hrsg.), Mutterschaft, Vaterschaft (S. 129-154). Weinheim: Beltz.

Hoffmann-Riem, C. (1989). Elternschaft ohne Verwandtschaft: Adoption, Stiefbeziehung und heterologe Insemination. In R. Nave-Herz \& M. Markefka (Hrsg.), Handbuch der Familien- und Jugendforschung, Bd. 1: Familienforschung (S 389-412). Neuwied: Luchterhand.

Maier, M. S. (2009). Homosexuelle Paare. In K. Lenz \& F. Nestmann (Hrsg.), Handbuch persönlicher Beziehungen (S. 259-276). Weinheim: Juventa.

Münder, J (2005). Familienrecht. München: Klüwer Deutschland GmbH. 
Nave-Herz, R. (2012). Familie heute - Wandel der Familienstrukturen und Folgen für die Erziehung. Darmstadt: Primus.

Rupp, M. (2009). Die Lebenssituation von Kindern in gleichgeschlechtlichen Lebenspartnerschaften. Köln: Bundesanzeiger.

Savin-Williams, R.C., \& Esterberg K. C. (2000). Lesbian, Gay and Bisexual Families. In D. H. Demo, K. R. Allen, \& M A. Fine (Hrsg.), Handbook of family diversity (S. 197-215). New York: Springer.

Steffens, C. (2010). Diskriminierung von Homo- und Bisexuellen. Aus Politik und Zeitgeschehen, $15 / 16,14-22$.

Streib-Brzic, U., \& Gerlach S. T. (2005). Und was sagen die Kinder dazu? Gespräche mit Töchtern und Söhnen lesbischer und schwuler Eltern. Berlin: Querverlag.

Tann v. d., C. (2012). Entwicklung in der Rechtsstellung eingetragener Lebenspartnerschaften. FamFR, 9, 195-199.

Vascovics, L. A., \& Buba H. P. (Hrsg.). (2000). Benachteiligung gleichgeschlechtlich orienter Personen und Paare. Studie im Auftrag des Bundesministerium der Justiz. Bonn: Nomos.

Wiesner, R. (2011). Kindeswohl. In Deutscher Verein für öffentliche und private Fürsorge e.V (Hrsg.), Fachlexikon soziale Arbeit (S. 515-516). Baden-Baden: Nomos. 